\title{
Sistemas de passeios aleatórios competitivos em $\mathrm{Z}$
}

\section{Mauricio Zuluaga Martinez}

\author{
TESE APRESENTADA \\ AO INSTITUTO DE MATEMÁTICA E ESTATÍSTICA \\ DA UNIVERSIDADE DE SÃO PAULO \\ PARA OBTENÇÃO \\ DO GRAU DE DOUTOR EM CIÊNCIAS
}

Área de Concentração: Estatística

Orientador: Prof. Dr. Fábio Prates Machado

São Paulo, 30 de março de 2007.

O autor agradece à FAPESP pelo apoio financeiro (Processo N. 02/07705-5). 


\title{
Sistemas de passeios aleatórios competitivos em $\mathrm{Z}$
}

\author{
Este exemplar corresponde à redação final \\ da tese devidamente corrigida \\ e defendida por Mauricio Zuluaga Martinez \\ e aprovada pela comissão julgadora.
}

São Paulo, 30 de março de 2007.

Banca examinadora:

Prof. Dr. Fábio Prates Machado (IME-USP)

Prof. Dr. Élcio Lebensztayn (IME-USP)

Prof. Dr. Rafael Andres Rosales Mitrowsky (FFCLRP-USP)

Prof. Dr. Marcio Argollo Ferreira de Menezes (UFF)

Prof. Dr. Klaus Leite Pinto Vasconcellos (UFPE) 
Para Samuel 


\section{Agradecimentos}

Agradeço ao meu orientador Prof. Dr. Fábio Prates Machado.

Também a Élcio Lebensztayn.

Agradeço aos meus colegas colombianos.

À FAPESP, pelo apoio recebido.

$\mathrm{E}$ a todos que me ajudaram a chegar até aqui. 


\section{Resumo}

Estudamos sistemas de passeios aleatórios sobre os vértices de um grafo conectado e infinito. Inicialmente há $N \geq 1$ partículas em cada vértice de $G^{\prime}$ um sub-

grafo de um grafo G, sendo todas inativas, exceto aquelas colocadas na "origem", um vértice escolhido do grafo. As partículas ativas realizam passeios aleatórios com tempo de vida $L$ sobre o conjunto dos vértices de G, movendo-se ao longo dos elos. Quando uma partícula ativa encontra uma inativa esta se ativa. Uma partícula ativa se extingue no instante em que completa $L$ saltos - consecutivos ou não - sem ativar nenhuma partícula.

Nosso objetivo é resolver algumas questões em aberto sobre estes processos, que podem ser considerados para modelar a dispersão de um vírus em uma rede de computadores.

Palavras-chave: modelo dos sapos, passeios aleatórios, processo de ramificação. 


\section{Abstract}

We study random walks systems on $Z$ whose general description follows. At time zero, there is a number $N \geq 1$ of particles at each vertex of $\mathbb{N}=\{1,2, \ldots\}$, all being inactive, except for those placed at the vertex one. Each active particle performs a simple random walk on $Z$ and, up to the time it dies, it activates all inactive particles that it meets along its way. An active particle dies at the instant it reaches a certain fixed total of jumps $(L \geq 1)$ without activating any particle. We investigate how the probability of survival of the process depends on $L$ and on the jumping probabilities of the active particles.

Let us give a motivation for the kind of model we work here. Assume that there is an infinite quantity of computers connected in line, and that each computer only communicates with the one on its right and the one on its left. Suppose that at time zero only one computer is infected by a virus which randomly chooses to jump to the computer on the left or the one on the right, infecting it. When one or more viruses (all viruses jump at discrete times) hit a computer, this computer is infected by a new virus which starts the same dynamics of jumps. After infected each computer activates an anti-virus which will kill any virus that jumps in the future on it. Is there a positive probability that an infinite quantity of computers will be infected? What happens if stronger virus are created, which are able to survive a larger number of computers with anti-virus?

Key words: frog model, random walk, Galton- Watson process, phase transition, epidemic model, frog model. 


\section{Sumário}

$\begin{array}{ll}\text { Motivação } & 1\end{array}$

1 Introdução 3

2 Modelo dos sapos 6

2.1 A Dinâmica das Partículas . . . . . . . . . . . . . . . 6

2.2 O Processo . . . . . . . . . . . . . . . . . . . . . . 7

$2.3 \mathrm{Um}$ Grafo particular . . . . . . . . . . . . . . . . . 8

2.4 O Processo em $G=\mathbb{Z} \ldots \ldots \ldots \ldots$

$\begin{array}{lll}3 & \text { O Processo Uniforme } & 12\end{array}$

3.1 Resultado mais importante: O Processo Uniforme com $G=G^{\prime}=\mathbb{Z}$ se Extingue q.c. . . . . . . . . . . . . . . . . . . . . . 14

3.2 Prova do Lema 3.1 e do Teorema $3.2 \ldots \ldots . . \ldots 15$

4 O Processo de Partícula $\quad 19$

4.1 O Processo de Partícula . . . . . . . . . . . . . . . . . . 20

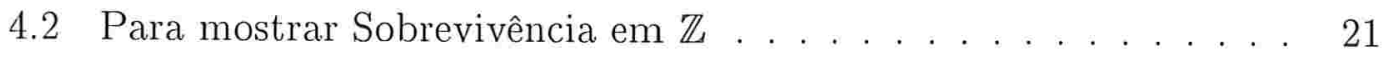

4.3 Prova do Teorema $4.2 \ldots \ldots . \ldots . \ldots . \ldots . . \ldots 22$

5 O Processo do Sítio 25

5.1 O Processo do Sítio . . . . . . . . . . . . . . . . 26

$\begin{array}{lr}\text { Referências Bibliográficas } & 29\end{array}$ 


\section{Motivação}

Uma quantidade infinita de computadores estão conectados em linha. Cada computador só se comunica com o de sua direita ou o de sua esquerda. No tempo $t=0 \mathrm{um}$ único computador é infectado com um vírus que faz um passeio aleatório, salta para o computador da esquerda ou da direita a tempo discreto até se extinguir. Quando um ou mais vírus (todos os vírus saltam no mesmo instante) atingem um computador o infectam com uma única réplica do vírus original que fará a mesma dinâmica de saltos. Cada computador após infectado ativa um antivírus que destruirá os vírus que pulem no futuro sobre ele . Existe probabilidade positiva de que uma quantidade indeterminada de computadores sejam infectados? Em outras palavras, em todo instante existe um computador com vírus?

Agora na rede anterior cada computador tem 1000 vírus inativos. No tempo $t=0$ os vírus de um único computador são ativados. Cada vírus pode saltar para o computador da esquerda ou da direita a tempo discreto até se extinguir. Quando um ou mais vírus atingem um computador ativam os 1000 vírus inativos nele. Cada computador após infectado ativa um antivírus que destruirá os vírus 
que pulem no futuro sobre ele . Em média a metade dos vírus pularão para a direita e a metade para a esquerda, logo em $t=1$ o computador à direita do inicialmente infectado teria 1500 vírus e em $t=2$ o da direita deste 1750 e assim por diante. Existe probabilidade positiva de que uma quantidade indeterminada de computadores sejam infectados? Em outras palavras, em todos os instantes existe um computador com vírus ativo?

Voltemos ao caso inicial. Agora o vírus pode ficar esperto: por exemplo pode ser que precise passar por dois ou mais computadores com antivírus para desaparecer, ou, sabendo da existência do antivírus, dar para seus descendentes velocidade de escape ou seja, dar maior probabilidade de saltar para a direita; a primeira geração $1-1 / 2$ a segunda $1-1 / 2^{2} \ldots$ ou ter mais descendentes; a primeira geração dois cada, a segunda três cada e assim por diante. Neste caso, existe probabilidade positiva de que uma quantidade indeterminada de computadores sejam infectados?

E no caso dos computadores com uma quantidade fixa de vírus inativos, existe uma quantidade, escolhida com antecipação, tal que com probabilidade positiva em todo instante exista algum computador com um vírus ativo? 


\section{Capítulo 1}

\section{Introdução}

Estudamos sistemas de passeios aleatórios sobre os vértices de um grafo conectado e infinito evoluindo a tempo discreto. Inicialmente há $N \geq 1$ partículas em cada vértice (ou sítio) de $G^{\prime}$, um subgrafo de um grafo $G$, sendo todas inativas, exceto aquelas colocadas na "origem", um vértice escolhido do grafo. Toda partícula ativa realiza um passeio aleatório sobre o conjunto dos vértices de G, movendo-se ao longo dos elos. Quando uma partícula ativa encontra partículas inativas estas se ativam. A seguir introduzimos um parâmetro para a extinção da uma partícula ativa: para $L \in\{1,2, \ldots\}$ fixado, uma partícula ativa se extingue no instante em que completa $L$ saltos - consecutivos ou não - sem ativar nenhuma partícula. Dizemos que o processo sobrevive, se para cada instante de tempo existe pelo menos uma partícula ativa.

A seguir elencamos alguns problemas estudados neste texto. Considere $\mathrm{G}^{\prime}=$ $\mathrm{G}=\mathbb{Z}$ ou $\mathrm{G}^{\prime}=\mathrm{G}=\mathbb{T}_{d}$ (árvore homogênea de grau $d+1$ ), $L \in\{1,2,3, \ldots\}$ e uma partícula, $N=1$, em cada vértice de $\mathrm{G}^{\prime}$. Existe transição de fase? Ou seja, existe 
um $L^{\prime} \in \mathbb{N}$ tal que o processo sobreviva para todo $L$ maior do que $L^{\prime}$ e o processo se extingue para todo $L$ menor ou igual a $L^{\prime}$ ?

A resposta é negativa caso $\mathrm{G}^{\prime}=\mathrm{G}=\mathbb{T}_{d}, d \geq 2$, pois para $L=1$ um processo de ramificação que sobrevive com probabilidade positiva é dominado pelo processo (o processo de ramificação tem sempre menos partículas ativas que as partículas ativas do processo)

Por outro lado para $L=1$ e $G^{\prime}=G=\mathbb{Z}$ podemos apresentar um processo de ramificação que se extingue q.c e domina o processo. E neste caso $\left(G^{\prime}=G=\mathbb{Z}\right)$ existe transição de fase? Respostas a essas perguntas e mais detalhes podem ser vistos no Capítulo 3.

Para o que vem a seguir consideremos $G=\mathbb{Z}$ e $G^{\prime}=\mathbb{N}$. Primeiramente suponha que $N=1$, isto é, temos uma partícula inativa em cada vértice de $\mathrm{G}^{\prime}=\mathbb{N}$ e uma ativa no sítio um, e que a partícula colocada originalmente na posição $n$, se ativada, salte sempre para a direita com probabilidade $P(1)=1-q_{n}$ e para a esquerda com probabilidade $P(-1)=q_{n}$, onde $0<q_{n}<1$. Se para algum $M \in\{1,2, \ldots\}$ temos que $\sum_{n=1}^{\infty}\left(q_{n}\right)^{M}<\infty$, então o processo tem probabilidade positiva de sobreviver com $L=1$ e também não temos, neste caso, transição de fase. Mais detalhes podem ser vistos no Capítulo 4 .

Coloquemos uma segunda dinâmica: Para $L \in\{1,2, \ldots\}$ fixado, uma partícula ativa se extingue no instante em que completa $L$ saltos - consecutivos ou não para a esquerda. É claro que se um processo se extingue q.c com a segunda 
dinâmica então o processo se extingue q.c. com a primeira.

Seja $G^{\prime}=G=\mathbb{Z}$ e consideremos a segunda dinâmica. Suponha que temos um número $N \geq 1$ fixo de partículas por vértice que se ativadas podem saltar para a direita com probabilidade $P(1)=p, 0<p<1$ fixo, e que todas as partículas à esquerda de um estão ativas. É possível mostrar que este processo se extingue q.c. para todo $L \in\{1,2, \ldots\}$ fixo, logo para este processo não temos transição de fase. Mais detalhes podem ser vistos no Capítulo 3.

Seja $K \geq 2$ inteiro par fixo. Consideremos $G=\mathbb{Z} \operatorname{com} G^{\prime}=\mathbb{N}$, uma partícula por sítio $(N=1)$, a partícula no sítio um acordada, as outras dormindo, e o seguinte: suponha que uma partícula ativada que está na posição $n$ salte para a direita com probabilidade $P(1)=1-q_{n}$ e para a esquerda com probabilidade $P(-1)=q_{n}$, onde $0 \leq q_{n}<1$ e se extingue quando completa $L$ saltos -consecutivos ou não-sem acordar ninguém. Se

$$
q_{n}= \begin{cases}(j+1)^{-2 /(K n)} & \text { se } n=s^{j} \text { onde } j \geq 1 \text { e } s>K+1 \\ 0 & \text { caso contrário }\end{cases}
$$

então o processo se extingue q.c. se e somente se $L \leq K$. Logo, neste processo temos transição de fase. Mais detalhes podem ser vistos no Capítulo 5. 


\section{Capítulo 2}

\section{Modelo dos sapos}

Vamos trabalhar com o modelo dos sapos, um sistema de passeios aleatórios em um grafo $G$, evoluindo a tempo discreto. As versões deste modelo estudadas até aqui têm uma diferença essencial dos modelos considerados neste trabalho: o tempo de vida de uma partícula ativa é ou infinito ou é uma variavel aleatória a qual é independente da trajetória da partícula. No primeiro caso o principal tema estudado é o teorema da forma em $\mathbb{Z}^{d}$, no segundo caso é investigar um parametro crítico em $\mathbb{Z}^{d}$ e em árvores homogêneas. Ver as referências bibliográficas.

\subsection{A Dinâmica das Partículas}

Sempre: no instante inicial as $N \geq 1$ partículas da "origem"são as únicas ativas. Cada partícula, depois de ativada, realiza um passeio aleatório e quando encontra partículas inativas estas se ativam.

Quando uma partícula ativa se extingue? Para $L \in\{1,2, \ldots\}$ fixado, uma partícula ativa se extingue no instante em que completa $L$ saltos - consecu- 
tivos ou não - sem ativar nenhuma partícula. Em outras palavras, cada partícula ativa começa com $L$ vidas e perde uma quando pula num vértice que já foi visitado pelo processo.

No caso $G=\mathbb{Z}$ podemos ter outras dinâmicas, por exemplo: uma partícula ativa se extingue no instante em que completa $L$ saltos - consecutivos ou não - para a esquerda. Mas se não falamos antes é porque a dinâmica é a de que: uma partícula ativa se extingue no instante em que completa $L, L \geq 1$, saltos consecutivos ou não - sem ativar nenhuma partícula.

O que pode mudar? A probabilidade de saltar sobre os vértices vizinhos. Tomamos essa probabilidade uniforme sobre os vértices, a menos que falemos o contrário. Se $G=\mathbb{Z}$ então $P(1)$ é a probabilidade de uma partícula ativa saltar para a direita e $P(-1)$ é a probabilidade de uma partícula ativa pular para a esquerda.

\subsection{O Processo}

Consideremos o modelo dos sapos em G com a dinâmica das partículas definida acima. No instante inicial, existe um número $N \geq 1$ de partículas em cada vértice de $G^{\prime} \subset \mathrm{G}$ mas só as partículas de um unico vértice estão acordadas. Queremos saber se o processo sobrevive, ou seja, se para cada instante existe pelo menos uma partícula ativa. Denotemos por $\mathbb{P}$ a medida de probabilidade deste processo. O seguinte lema será útil (ver Bremaud [13, p. 422]) 
Lema 2.1 Seja $0<a_{n}<1$. Então

$$
\prod_{n=1}^{\infty}\left(1-a_{n}\right)=0 \Longleftrightarrow \sum_{n=1}^{\infty} a_{n}=\infty .
$$

Prova Usando $1-a_{k} \leq \exp \left(-a_{k}\right)$ obtemos $0 \leq \prod_{n=1}^{\infty}\left(1-a_{n}\right) \leq \exp \left(-\sum_{n=1}^{\infty} a_{n}\right)$ portanto se $\sum_{n=1}^{\infty} a_{n}=\infty$ então $\prod_{n=1}^{\infty}\left(1-a_{n}\right)=0$.

Agora se $\sum_{n=1}^{\infty} a_{n}<\infty$ então $\sum_{n=m+1}^{\infty} a_{n}<0.1$ para algum $m \in \mathbb{N}$. Logo $\prod_{n=1}^{\infty}\left(1-a_{n}\right)=\prod_{n=1}^{m}\left(1-a_{n}\right) \prod_{n=m+1}^{\infty}\left(1-a_{n}\right) \geq \prod_{n=1}^{m}\left(1-a_{n}\right)\left(1-\sum_{n=m+1}^{\infty} a_{n}\right)>$ $\prod_{n=1}^{m}\left(1-a_{n}\right) 0.9>0$.

\subsection{Um Grafo particular}

A seguir veremos um processo com $G^{\prime}=G=\mathbb{Z}, L=N=1$ e $P(1)=$ $P(-1)=1 / 2$. Neste caso um processo de ramificação que se extingue q.c domina o processo que estamos estudando.

Lema $2.2 S e \mathrm{G}^{\prime}=\mathrm{G}=\mathbb{Z}, L=N=1$ e $P(1)=P(-1)=1 / 2$ então o processo se extingue q.c.

uma partícula por vértice, $N=1$

as partículas ativas têm uma vida, $L=1$.

$$
\frac{1}{2}
$$


Prova : Em cada instante, o número de partículas no processo é menor ou igual à população do processo de ramificação em que um indivíduo tem zero ou dois descendentes, a partícula que acordou e ele mesmo, com probabilidade $\frac{1}{2}$. Como este processo se extingue q.c., o mesmo ocorre com o nosso processo.

Para uma generalização do Lema 2.2 ver Lema 3.1.

O processo que queremos estudar tem comportamento diferente dependendo do grafo: veremos por exemplo que no caso $L=N=1$ e $\mathrm{G}^{\prime}=\mathrm{G}=\mathbb{T}_{d}$ o processo tem probabilidade positiva de sobreviver mas no caso $G^{\prime}=G=\mathbb{Z}$ o processo se extingue q.c.

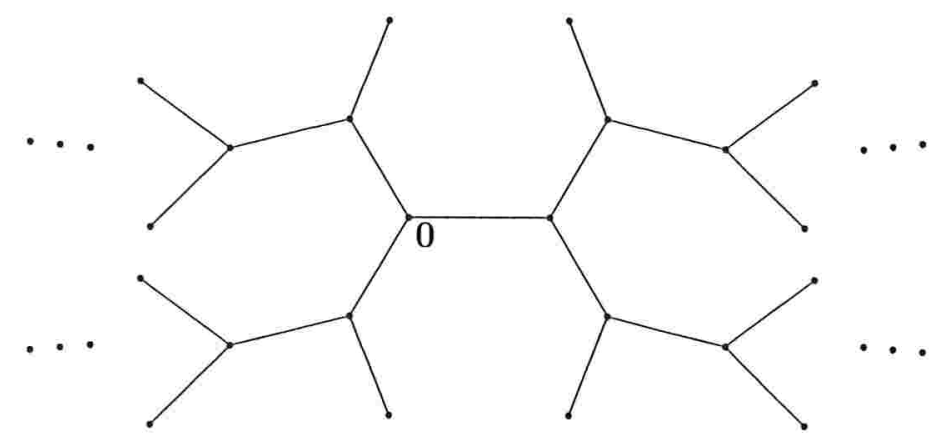

Figura 2.1: Uma representação de $\mathbb{T}_{2}$, a árvore homogênea de grau 3.

\section{O Processo em $\mathbb{T}_{d}$, a Árvore Homogênea de Grau $d+1$}

Lema 2.3 Se $L=N=1$ e $\mathrm{G}^{\prime}=\mathrm{G}=\mathbb{T}_{d}$, a árvore homogênea de grau $d+1$, $d \geq 2$, então o processo tem probabilidade positiva de sobreviver.

Prova : Vamos definir um processo de ramificação cuja sobrevivência implica a sobrevivência do processo estudado. Definamos superpartícula ao conjunto 
de duas partículas acordadas num mesmo vértice de $\mathbb{T}_{d}$. Quando tivermos mais de duas partículas acordadas num mesmo vértice eliminamos o excesso para que fiquem só duas. Observemos então que a probabilidade de uma superpartícula ter zero filhos é $\frac{1}{(d+1)} \cdot \frac{1}{(d+1)}$, um filho é $2 \frac{d}{(d+1)} \cdot \frac{1}{(d+1)}+\frac{d}{(d+1)} \cdot \frac{1}{(d+1)}$, dois filhos é $\frac{d}{(d+1)} \cdot \frac{(d-1)}{(d+1)}$ logo a média do número de filhos é $\frac{\left(2 d^{2}+d\right)}{(d+1)^{2}}>1$ se $d \geq 2$. Isto prova que o processo na árvore sobrevive com probabilidade positiva pois ele domina o processo de ramificação recém-definido.

\subsection{O Processo em $G=\mathbb{Z}$}

O grafo $G=\mathbb{Z}$ é estudado neste trabalho, a seguir apresentamos alguns resultados que obtivemos neste caso.

Consideremos o modelo dos sapos em $G=\mathbb{Z} \operatorname{com} G^{\prime}=\mathbb{N}$. Suponha que a partícula na posição $n$, se ativada, salte para a direita com probabilidade $P(1)=$ $1-q_{n}$ e para a esquerda com probabilidade $P(-1)=q_{n}$, onde $0<q_{n}<1$ para todo $n$.

Vamos descrever três modelos e estabelecer seus resultados. O fato unificador é $L$ que é o número de vidas que cada partícula ativa tem, significando o número de visitas que ela pode fazer a vértices ja visitados antes de se extinguir.

O Processo Uniforme: Fixemos $L \geq 1, N \geq 1$ inteiros e $0<p<1$ real. Inicialmente há $N$ partículas em cada vértice de $G^{\prime}=\mathbb{N}$ sendo todas inativas, exceto aquelas colocadas no sítio um. Toda partícula ativa realiza um passeio 
aleatório sobre o conjunto dos vértices de $G=\mathbb{Z}$, movendo-se para a direita com probabilidade $P(1)=p$ e para a esquerda com probabilidade $P(-1)=1-p$. O Processo uniforme morre q.c. Mais detalhes podem ser vistos no Capítulo 3.

O Processo de Partícula: Inicialmente há uma partícula em cada vértice de $G^{\prime}=\mathbb{N}(N=1)$, sendo todas inativas, exceto aquela colocada no sítio um. Suponha que a partícula originalmente na posição $n$, se ativada, salte sempre para a direita com probabilidade $P(1)=1-q_{n}$ e para a esquerda com probabilidade $P(-1)=q_{n}$, onde $0<q_{n}<1$. Se para algum $M \in\{1,2, \ldots\}$ temos que $\sum_{n=1}^{\infty}\left(q_{n}\right)^{M}<\infty$, então o Processo de partícula tem probabilidade positiva de sobreviver com $L=1$. Mais detalhes podem ser vistos no Capítulo 4.

O Processo do Sítio: Seja $K \geq 2$ inteiro par fixo. Consideremos uma partícula por sítio em $\mathrm{G}^{\prime}=\mathbb{N}(N=1)$, a partícula no sítio um acordada, as outras dormindo e o seguinte: suponha que uma partícula ativada que está na posição $n$ salte para a direita com probabilidade $P(1)=1-q_{n}$ e para a esquerda com probabilidade $P(-1)=q_{n}$, e desaparece quando dá $L$ saltos -consecutivos ou não-sem acordar ninguém. Seja

$$
q_{n}= \begin{cases}(j+1)^{-2 /(K n)} & \text { se } n=s^{j} \text { onde } j \geq 1 \text { e } s>K+1 \\ 0 & \text { caso contrário. }\end{cases}
$$

Então o Processo do sítio morre q.c. se e somente se $L \leq K$. Mais detalhes podem ser vistos no Capítulo 5. 


\section{Capítulo 3}

\section{O Processo Uniforme}

Uma partícula ao realizar um passeio aleatório simples simétrico em $G=\mathbb{Z}$ visita todos os vértices q.c. Fixe $L \in\{1,2, \ldots\}$ bem grande. O que ocorre se em cada vértice de $\mathrm{G}^{\prime}=\mathbb{Z}$ for colocada uma partícula inativa $(N=1)$ que pode pular $L$ vezes ao ser acordada? Em $t=0$ ative a partícula no sítio um. Considere a seguinte dinâmica: quando uma partícula ativa pula (todas as partículas ativas pulam no mesmo instante) sobre uma partícula inativa, esta fica ativada e pulará $L$ vezes e depois desaparecerá. Há probabilidade positiva de se ter em todo instante alguma partícula ativa? A resposta é não. Isto é consequiência do resultado mais importante deste trabalho, o Teorema 3.2.

\section{O Processo Uniforme em $\mathbb{N}$}

Fixe inteiros $L \geq 1, N \geq 1$ e $0<p<1$ real. Suponha que temos uma quantidade infinita de computadores conectados em linha. Cada computador só se comunica com o vizinho à direita ou à esquerda. Um vírus é criado. Este pode saltar, a tempo discreto, para a esquerda com probabilidade $P(-1)=1-p$ e para 
a direita com probabilidade $P(1)=p$. Quando o vírus atinge um computador este ativa um antivírus. Um computador com antivírus ativado pode destruir vírus que ali pularem. Suponha que o vírus sobrevive a muitas visitas a computadores com antivírus ativados, isto é, $L$ é muito grande. $O$ criador do vírus coloca $N$ ( $N$ muito grande) réplicas deste em cada computador, todos inativos. No tempo $t=0$ todos os vírus no sítio um são ativados. Quando um ou mais vírus (todos os vírus saltam no mesmo instante) atingem um computador, os vírus inativos ali presentes são ativados e passam a fazer a mesma dinâmica. Dizemos que o processo sobrevive, se para cada instante de tempo existe pelo menos um vírus ativo. Existe probabilidade positiva de que uma quantidade indefinida de computadores sejam infectados? Em outras palavras, o Processo uniforme sobrevive com probabilidade positiva?

O Processo Uniforme: $G=\mathbb{Z}, G^{\prime}=\mathbb{N}$

$N$ partículas por vértice de $\mathrm{G}^{\prime}=\mathbb{N}$

cada partícula ativa com $L$ vidas

$1-p$

$p$

$N, L \in \mathbb{N}, p \in(0,1)$ fixos.

Lema 3.1 O Processo uniforme se extingue q.c. Isto é quando temos $\mathrm{G}=\mathbb{Z}$, 
$\mathrm{G}^{\prime}=\mathbb{N}, L \geq 1, N \geq 1$ inteiros fixos e $0<p<1$ real fixo tal $P(1)=p e$ $P(-1)=1-p$, em algum instante (aleatorio finito) não teremos vírus ativo, com probabilidade um.

A demonstração do Lema 3.1 é apresentada na Seção 3.2

A seguir obteremos uma generalização do Lema 3.1 que nos diz que em $\mathrm{G}=$ $\mathrm{G}^{\prime}=\mathbb{Z}$ não importa o número de partículas por sítio nem a probabilidade de saltar para a direita, sempre que estas sejam constantes e iguais para cada vértice, o resultado é o mesmo: o processo se extingue q.c.

\subsection{Resultado mais importante: O Processo Uni- forme com $G=G^{\prime}=\mathbb{Z}$ se Extingue q.c.}

O processo uniforme em $\mathbb{Z}$. Consideremos o modelo dos sapos com $G=$ $\mathrm{G}^{\prime}=\mathbb{Z}$ e a dinâmica usual: para $N, L \in \mathbb{N}$ fixados, no instante inicial, existem $N$ partículas em cada vértice de $G^{\prime}=\mathbb{Z}$, todas inativas exceto as do sítio um; cada partícula começa com $L$ vidas e perde uma quando pula num vértice que já foi visitado. $P(1)=p$ e $P(-1)=1-p$, onde $p \in(0,1)$ fixo, são as probabilidades de uma partícula ativa pular à direita ou esquerda respectivamente e as partículas se ativen quando são visitadas por partículas ativas. Então, generalizando o Lema 3.1:

Teorema 3.2 O processo uniforme em $\mathbb{Z}$ se extingue q.c. 
O Processo Uniforme Em $\mathbb{Z}$

$N$ partículas em cada vértice de $\mathbb{Z}$

Cada partícula ativa com $L$ vidas

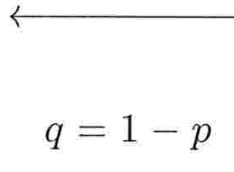

$$
\begin{gathered}
\cdots \cdots \\
N, L \in \mathbb{N}, p \in(0,1) \text { fixos. }
\end{gathered}
$$$$
q=1-p
$$

A prova do Teorema 3.2 é encontrada na Seção 3.2.

Problema 1 Se $\mathrm{G}^{\prime}=\mathrm{G}=\mathbb{Z}^{d}, d \geq 2$ e $N=L=1$. Então o processo se extingue q.c. ou o processo tem probabilidade positiva de sobreviver?

Agora começamos a fazer as provas dos resultados enunciados acima.

\subsection{Prova do Lema 3.1 e do Teorema 3.2}

Para provar o Lema 3.1 consideramos o Processo uniforme independente: uma partícula ativa perde uma de suas $L$ vidas sempre que ela pula em direção à origem e então a vida de uma partícula ativa depende de seu próprio passeio aleatório independente de todos os outros passeios. $G=\mathbb{Z}, G^{\prime}=\mathbb{N}, N, P(1)$ e $P(-1)$ como no processo uniforme. É claro que o processo uniforme independente domina o processo uniforme.

Lema 3.3 O processo uniforme independente se extingue q.c. 
Prova Para $i, j \in \mathbb{N}$ definamos os eventos $A_{i}:=$ as $N$ partículas no sítio $i$ são acordadas, $\{i \rightarrow j\}:=$ alguma das $N$ partículas no vértice $i$ visita o vértice $j$ e $\{i \nrightarrow j\}:=\{i \rightarrow j\}^{c}$. Também definamos

$$
B_{i}=\bigcup_{j=1}^{i-1}\{j \rightarrow 2 i\}
$$

e

$$
C_{i}=\bigcup_{j=1}^{2 i-1}\{j \rightarrow 2 i\}
$$

Notemos que

$$
A_{2 i} \subset A_{i} \cap\left\{B_{i} \cup C_{i}\right\} \subset B_{i} \cup\left\{A_{i} \cap C_{i}\right\}
$$

e pela independência do Processo uniforme independente temos que

$$
\mathbb{P}\left(A_{2 i}\right) \leq \mathbb{P}\left(B_{i}\right)+\mathbb{P}\left(A_{i}\right) \mathbb{P}\left(C_{i}\right)
$$

Como

$$
\mathbb{P}(j \rightarrow 2 i)=\mathbb{P}(j \rightarrow j+1) \prod_{k=j+1}^{2 i-1} \mathbb{P}(j \rightarrow k+1 \mid j \rightarrow k) \leq(\mathbb{P}(1 \rightarrow 2))^{2 i-j}
$$

para todo $1<j<2 i$. Temos, para $c=(1-\mathbb{P}(1 \rightarrow 2))^{-1}$, que

$$
\mathbb{P}\left(B_{i}\right) \leq \sum_{j=1}^{i-1} \mathbb{P}(j \rightarrow 2 i)<c(\mathbb{P}(1 \rightarrow 2))^{i}
$$

onde $\mathbb{P}(1 \rightarrow 2) \leq 1-(1-p)^{L N}<1$. Também 


$$
\begin{aligned}
\mathbb{P}\left(C_{i}\right) & =1-\mathbb{P}\left(C_{i}^{c}\right) \\
& =1-\mathbb{P}\left(\bigcap_{j=i}^{2 i-1}\{j \not \neg 2 i\}\right) \\
& =1-\mathbb{P}\left(\bigcap_{j=2}^{i+1}\{1 \not \neg j\}\right) \\
& =1-\prod_{j=2}^{i+1} \mathbb{P}(1 \not \neg j) \\
& \leq 1-\prod_{j=2}^{\infty} \mathbb{P}(1 \not \neg j) \\
& =1-\prod_{j=2}^{\infty}(1-\mathbb{P}(1 \rightarrow j))
\end{aligned}
$$

e usando o Lema 2.1, pois $\sum_{j=2}^{\infty} \mathbb{P}(1 \rightarrow j)<\sum_{j=2}^{\infty} \mathbb{P}(1 \rightarrow 2)^{j-1}<\infty$, obtemos que para algum $0<r<1$

$$
\mathbb{P}\left(C_{i}\right)<r \text { para todo } i \text {. }
$$

Como $r>\mathbb{P}(1 \rightarrow 2)$, temos, de (3.1), (3.2) e (3.3), temos que

$$
\mathbb{P}\left(A_{2 i}\right) \leq c r^{i}+r \mathbb{P}\left(A_{i}\right)
$$

Assim

$$
\mathbb{P}\left(A_{2^{j}}\right) \leq c r^{2^{j-1}}+r \mathbb{P}\left(A_{2^{j-1}}\right)
$$

e então

$$
\mathbb{P}\left(A_{2^{j}}\right)<c j r^{j-1} \longrightarrow 0 \text { quando } j \longrightarrow \infty
$$

Agora, dado $n$ existe um único $j=j(n)$, tal que $2^{j} \leq n<2^{j+1}$ e, como

$$
\mathbb{P}\left(A_{n}\right) \leq \mathbb{P}\left(A_{2^{j}}\right)
$$


temos que

$$
\mathbb{P}\left(A_{n}\right) \longrightarrow 0 \text { quando } n \longrightarrow \infty \text {. }
$$

Para terminar a prova, observemos que

$$
\begin{aligned}
\mathbb{P}(\text { sobreviver }) & =\mathbb{P}\left(A_{2} \cap A_{3} \cap \ldots \cap A_{n} \cap \ldots\right) \\
& =\lim _{n \rightarrow \infty} \mathbb{P}\left(A_{n}\right) \\
& =0
\end{aligned}
$$

Logo, também, o processo uniforme se extingue q.c, pois o processo uniforme independente domina o processo uniforme. Como veremos a seguir, uma adaptação da prova do Lema 3.3 nos permitirá demonstrar o Teorema 3.2.

Prova do Teorema 3.2. Consideremos as notações introduzidas na prova do Lema 3.3, porém fazemos $B_{i}=\bigcup_{j=-\infty}^{i-1}\{j \rightarrow 2 i\}$. Observe que ainda assim vale a Equação (3.2), com o mesmo $c$, portanto o mesmo argumento leva à conclusão de que

$$
\lim _{n \rightarrow \infty} \mathbb{P}\left(A_{n}\right)=0
$$

Analogamente, temos que $\lim _{n \rightarrow \infty} \mathbb{P}\left(A_{-n}\right)=0$. Daí segue que o processo em $\mathbb{Z}$ se extingue q.c.

Observemos que podemos assumir que todas as partículas à esquerda de um estão acordadas no instante inicial; a prova não muda, $B_{i}=\bigcup_{j=-\infty}^{i-1}\{j \rightarrow 2 i\}$, de novo. 


\section{Capítulo 4}

\section{O Processo de Partícula}

Sejam $G=\mathbb{Z}, G^{\prime}=\mathbb{N}$ e fixe $L=N=1$. O criador do vírus cria agora, um vírus mais fraco $(L=1)$. Entretanto, a velocidade de escape do vírus é maior: a partícula (vírus) acordada no sítio $n$ pulará sempre para a direita com probabilidade $P(1)=1-q_{n}$ onde $q_{n}$ vai para zero quando $n$ vai para infinito. Este vírus evoluido é mais caro e só é possível colocar uma $(N=1)$ cópia por computador (lembre-se de que há um computador em cada sítio de $G^{\prime}=\mathbb{N}$ ). No tempo $t=0$ o vírus no sítio um é ativado. Quando um ou mais vírus (todos os vírus saltam no mesmo instante) atingem um computador, o vírus inativo ali presente é ativado e passa a realizar a mesma dinâmica. Quando um vírus atinge um computador, este ativa um antivírus que destruirá os vírus que pularem sobre ele no futuro. Dizemos que o processo sobrevive, se para cada instante de tempo existe pelo menos um vírus ativo. Existe probabilidade positiva de que uma quantidade indefinida de computadores sejam infectados? Em outras palavras, o Processo de Partícula sobrevive com probabilidade positiva? 


\subsection{O Processo de Partícula}

Consideremos o modelo dos sapos em $G=\mathbb{Z}$ com $G^{\prime}=\mathbb{N}$ e o seguinte: suponha uma partícula por vértice $(N=1)$, e que a partícula na posição $n$, quando ativada, salte sempre para a direita com probabilidade $P(1)=1-q_{n}$ e para a esquerda com probabilidade $P(-1)=q_{n}$, onde $0<q_{n}<1$ para todo $n$. As partículas ativas desaparecem inmediatamente após pular para um vértice ja visitado, ou seja, quando pulam para a esquerda $(L=1)$. Denominamos este O Processo de partícula.

Uma conseqüência imediata do Teorema 3.2 é:

Corolário 4.1 Se $\inf _{n} q_{n}>0$ então o processo de partícula se extingue q.c.

O principal resultado deste capítulo apresenta uma condição sob a qual o processo sobrevive com probabilidade positiva.

Teorema 4.2 Se $\sum_{n=1}^{\infty} q_{n} q_{n+1} \ldots q_{n+M-1}<\infty$ para algum $M \in\{1,2, \ldots\}$, então o processo de particula tem probabilidade positiva de sobreviver.

Como $\sum_{n=1}^{\infty} \prod_{i=0}^{M-1} q_{n+i} \leq M \sum_{n=1}^{\infty}\left(q_{n}\right)^{M}$, temos que:

Corolário 4.3 Se $\sum_{n=1}^{\infty}\left(q_{n}\right)^{M}<\infty$ para algum $M \in\{1,2, \ldots\}$, então o processo de particula tem probabilidade positiva de sobreviver.

Logo, se $\left(q_{1}, q_{2}, \ldots\right)$ é uma permutação de $\left(\left(\frac{1}{2}\right)^{\eta},\left(\frac{1}{3}\right)^{\eta},\left(\frac{1}{4}\right)^{\eta}, \ldots\right)$, onde $0<\eta \leq 1$ fixo, então o processo de partícula tem probabilidade positiva de sobreviver. 
O Processo De Partícula

uma partícula por vértice, $N=1$

as partículas ativas têm uma vida, $L=1$

$q_{n}$

$$
p_{n}=1-q_{n} .
$$

$$
\begin{gathered}
M \in\{1,2,3, \ldots\} \text { fixo e } \\
\sum_{n=1}^{\infty} q_{n} q_{n+1} \ldots q_{n+M-1}<\infty .
\end{gathered}
$$

\subsection{Para mostrar Sobrevivência em $\mathbb{Z}$}

O seguinte resultado será útil para obter sobrevivência em $\mathbb{Z}$, ver prova do Teorema 4.2. Para $n \in \mathbb{N}$ definamos os eventos: $A_{n}:=$ a partícula no sítio $n$ é acordada, $\{n \rightarrow m\}:=$ a partícula no vértice $n$ visita o vértice $m$ e $\{n \nrightarrow m\}:=$ $\{n \rightarrow m\}^{c}$. Então

$$
A_{n+1} \supset\left\{A_{n}, n \rightarrow n+1\right\} \dot{\cup}\left\{A_{n-1}, n-1 \rightarrow n+1, n \nrightarrow n+1\right\} .
$$

Como $L=1$ implica independência dos eventos, teremos o seguinte lema que será útil para mostrar sobrevivência quando $M=1$ e $M=2$ :

Lema 4.4 (a) $\mathbb{P}\left(A_{n}\right) \mathbb{P}(n \rightarrow n+1) \leq \mathbb{P}\left(A_{n+1}\right)$.

(b) $\mathbb{P}\left(A_{n}\right)[\mathbb{P}(n-1 \rightarrow n+1) \mathbb{P}(n \nrightarrow n+1)+\mathbb{P}(n \rightarrow n+1)] \leq \mathbb{P}\left(A_{n+1}\right)$. 
Lembremos que $0<q_{n}<1$ no processo de partícula e observemos que se o infimo dos $q_{n}$ é positivo então o processo se extingue q.c. Pois se $q$ é o infimo dos $q_{n}$, pelo Teorema 3.2, o processo com uma partícula por sítio e probabilidade de saltar para a direita $p=1-q$ morre q.c, então, por comparação, o processo de partícula se extingue q.c.

Problema 2 Que acontece com os diferentes processos se $P(-1)=q_{n}$ e o infimo dos $q_{n}$ é zero?

A seguir apresentamos a prova do resultado enunciado acima.

\subsection{Prova do Teorema 4.2}

Prova do Teorema 4.2: caso $M=1$ Pelo Lema 4.4 parte (a) temos que

$$
\mathbb{P}\left(A_{n}\right)\left[1-q_{n}\right] \leq \mathbb{P}\left(A_{n+1}\right) .
$$

Portanto

$$
\mathbb{P}\left(A_{n+1}\right) \geq\left(1-q_{1}\right)\left(1-q_{2}\right) \cdots\left(1-q_{n}\right) .
$$

Agora pelo Lema 2.1

$$
\left(1-q_{1}\right)\left(1-q_{2}\right) \cdots\left(1-q_{n}\right) \cdots \neq 0 .
$$

Logo, a probabilidade de sobreviver não é zero, pois

$$
\mathbb{P}(\text { sobreviver })=\lim _{n \rightarrow \infty} \mathbb{P}\left(A_{n+1}\right)
$$


Prova do Teorema 4.2: caso $M=2$. Pelo Lema 4.4 parte $(b)$ temos que

$$
\begin{aligned}
\mathbb{P}\left(A_{n+1}\right) & \geq \mathbb{P}\left(A_{n}\right)\left[\left(1-q_{n-1}\right)^{2} q_{n}+\left(1-q_{n}\right)\right] \\
& \geq \mathbb{P}\left(A_{n}\right)\left[1-2 q_{n-1} q_{n}\right]
\end{aligned}
$$

logo, pelo Lema 2.1, a probabilidade de sobreviver não é zero, pois:

$$
\left(1-2 q_{1} q_{2}\right)\left(1-2 q_{2} q_{3}\right) \cdots\left(1-2 q_{n-1} q_{n}\right) \cdots \neq 0
$$

Prova do Teorema 4.2 caso geral $(M \geq 1)$. A idéia é a mesma dos casos $M=1$ e $M=2$. Tomemos $n>M$ e lembremos que se $0<q<1$ então para todo $j \in \mathbb{N}$ temos que

$$
(1-q)^{j}>1-j q
$$

portanto para $0<q_{j}<1$ temos que

$$
\prod_{j=n-M+1}^{n} 1-\left(1-q_{j}\right)^{n+1-j}<M ! \prod_{j=n-M+1}^{n} q_{j}
$$

Agora

$$
\begin{aligned}
A_{n+1} \supset\left(A_{n} \cap\{n \rightarrow n+1\}\right) \cup & \\
\bigcup_{i=n-M+1}^{n-1} & \left(A_{i} \cap\{i \rightarrow n+1\} \cap \bigcap_{j=i+1}^{n}\{j \nrightarrow n+1\}\right) .
\end{aligned}
$$

Assim 


$$
\begin{aligned}
\mathbb{P}\left(A_{n+1}\right) & \geq \mathbb{P}\left(A_{n}\right)\left[\mathbb{P}(n \rightarrow n+1)+\sum_{i=n-M+1}^{n-1} \mathbb{P}(i \rightarrow n+1) \prod_{j=i+1}^{n} \mathbb{P}(j \nrightarrow n+1)\right] \\
& =\mathbb{P}\left(A_{n}\right)\left[1-\prod_{i=n-M+1}^{n} \mathbb{P}(i \nrightarrow n+1)\right] \\
& =\mathbb{P}\left(A_{n}\right)\left[1-\prod_{i=n-M+1}^{n} 1-\left(1-q_{i}\right)^{n+1-i}\right] \\
& \geq \mathbb{P}\left(A_{n}\right)\left[1-\prod_{i=n-M+1}^{n}(n+1-i) q_{i}\right] \\
& =\mathbb{P}\left(A_{n}\right)\left[1-M ! \prod_{i=n-M+1}^{n} q_{i}\right] .
\end{aligned}
$$

logo, pelo Lema 2.1 , temos para $t$ suficientemente grande que

$$
\mathbb{P}\left(A_{n+t}\right) \geq C \mathbb{P}\left(A_{t}\right)
$$

onde $C>0$ não depende de $n$. Fazendo $n \rightarrow \infty$ o resultado se segue. 


\section{Capítulo 5}

\section{O Processo do Sítio}

Sejam $\mathrm{G}=\mathbb{Z}, \mathrm{G}^{\prime}=\mathbb{N}$ e fixemos $N=1$. O placar está 1-1: os vírus morrem q.c no processo uniforme mas sobrevivem com probabilidade positiva no processo de partícula. Agora os vírus sabem o que têm que fazer: pular para a direita, $P(1)=1$. Mas os computadores têm uma nova arma, além de ativar o antivírus quando são visitados, eles colocam muros espalhados a distâncias pré-escolhidas que empurram os vírus para a esquerda e quando isto acontece os vírus perdem duas vidas, pois isto significa visitar dois computadores com antivírus. As barreiras $q_{n}$ dependem de $K$, um inteiro par que o criador do vírus não conhece, e cada vez são mais altas: empurram os vírus para a esquerda com probabilidade que vai para um com $n$. Os vírus atingem os muros como um sunami: chegam a estes em grupos muito grandes e, se algum deles consegue pular a barreira, ativa um grande número de vírus que chegarão até o próximo obstáculo. Para que isto aconteça, o criador do vírus deverá dar a este um número de vidas adequado. Ele escolhe dar a seu vírus $L$ vidas e $P(1)=1$, esta probabilidade não funciona nos 
sítios com barreira, e coloca uma cópia dele por computador, $N=1$, em cada sítio de $\mathrm{G}^{\prime}=\mathbb{N}$. No tempo $t=0$, o vírus no sítio um é ativado e quando um ou mais vírus (todos os vírus saltam no mesmo instante) atingem um computador, o vírus ali localizado é ativado e passa a realizar a mesma dinâmica. Quando um vírus atinge um computador este ativa um antivírus que pode destruir os vírus que pulem sobre ele no futuro. Dizemos que o processo sobrevive, se para cada instante de tempo existe pelo menos um vírus ativo. Será que o processo sobrevive?

\subsection{O Processo do Sítio}

Seja $K \in\{2,4,6, \ldots\}$. Consideremos o modelo dos sapos em $G=\mathbb{Z} \operatorname{com} G^{\prime}=\mathbb{N}$ e o seguinte: suponha uma partícula por vértice $(N=1)$, e que a partícula que atinja a posição $n$ salte para a direita com probabilidade $P(1)=1-q_{n}$ e para a esquerda com probabilidade $P(-1)=q_{n}$, onde

$$
q_{n}= \begin{cases}(j+1)^{-2 /(K n)} & \text { se } n=s^{j} \text { onde } j \geq 1 \text { e } s \in \mathbb{N} \text { é tal que } K+1<s \\ 0 & \text { caso contrário. }\end{cases}
$$

As partículas ativas têm $L$ vidas e perdem uma após pular para um vértice já visitado. Denominamos este como O Processo do sítio.

Na prova do seguente teorema nos aproveitaremos de um fato conhecido:

$$
\sum_{n=1}^{\infty} 1 / n^{t}= \begin{cases}\infty & \text { se } t \leq 1 \\ <\infty & \text { se } t>1\end{cases}
$$

Teorema 5.1 O processo do sitio se extingue q.c. se e somente se $L \leq K$. 


\section{O Processo Do Sitio}

uma partícula por vértice, $N=1$

uma partícula ativa tem $L$ vidas

$q_{n}$

$$
p=1-q_{n}
$$

$q_{n}:=$ probabilidade da partícula que

atinga o sítio $n$ saltar para esquerda

$$
\begin{gathered}
q_{n}=(j+1)^{\frac{-1}{K_{n}}} \text { se } n=s^{j}, K+1<s \\
q_{n}=0 \text { c.c. }
\end{gathered}
$$

\section{Prova :}

Temos que provar duas afirmações.

(i) O processo se exingue q.c se $L=K$.

(ii) O processo sobreveve com probabilidade positiva se $L=K+1$.

Para $j \geq 1$ seja $A_{j}$ o evento que a partícula no vértice $s^{j}$ é ativada

Vejamos primeiro que o processo se extingue para $L=K$. Observemos que neste caso temos no máximo $s^{j}$ partículas no vértice $s^{j}$, cada uma com no máximo 
$L$ vidas. Agora

$$
\begin{aligned}
\mathbb{P}\left(A_{j+1}\right) & =\mathbb{P}\left(A_{1}, A_{2}, \ldots, A_{j}, A_{j+1}\right) \\
& =\mathbb{P}\left(A_{1}\right) \mathbb{P}\left(A_{2} \mid A_{1}\right) \ldots \mathbb{P}\left(A_{j+1} \mid A_{j}\right) \\
& \leq\left(1-\left(\left(q_{s^{1}}\right)^{K / 2}\right)^{s^{1}}\right)\left(1-\left(\left(q_{s^{2}}\right)^{K / 2}\right)^{s^{2}}\right) \cdots\left(1-\left(\left(q_{s^{j}}\right)^{K / 2}\right)^{s^{j}}\right),
\end{aligned}
$$

temos, pelo lema 2.1, que a probabilidade de sobreviver é zero, pois

$$
\begin{gathered}
\sum_{j=1}^{\infty}\left(\left(q_{s^{j}}\right)^{K / 2}\right)^{s^{j}}=\sum_{j=1}^{\infty}(j+1)^{-1}=\infty, \mathrm{e} \\
\mathbb{P}(\text { sobreviver })=\lim _{j \rightarrow \infty} \mathbb{P}\left(A_{j}\right)
\end{gathered}
$$

Agora vejamos que o processo sobrevive com probabilidade positiva para $L=$ $k+1$. Observemos que se acontece $A_{j}$ temos no minimo $s^{j}-s^{j-1}+1$ partículas no vértice $s^{j} \operatorname{com} L$ vidas cada uma. Agora

$$
\begin{aligned}
\mathbb{P}\left(A_{j+1}\right) & =\mathbb{P}\left(A_{1}, A_{2}, \ldots, A_{j}, A_{j+1}\right) \\
& =\mathbb{P}\left(A_{1}\right) \mathbb{P}\left(A_{2} \mid A_{1}\right) \ldots \mathbb{P}\left(A_{j+1} \mid A_{j}\right) \\
& \geq\left(1-\left(\left(q_{s^{1}}\right)^{K / 2+1}\right)^{s^{1}}\right)\left(1-\left(\left(q_{s^{2}}\right)^{K / 2+1}\right)^{s^{2}-s^{1}+1}\right) \cdots\left(1-\left(\left(q_{s^{j}}\right)^{K / 2+1}\right)^{s^{j}-s^{j-1}+1}\right),
\end{aligned}
$$

temos, pelo lema 2.1, que a probabilidade de sobreviver não é zero, pois

$$
\sum_{j=2}^{\infty}\left(\left(q_{s^{j}}\right)^{K / 2+1}\right)^{s^{j}-s^{j-1}+1}<\sum_{j=1}^{\infty}(j+1)^{-a}<\infty,
$$

ande $a:=\left(1-s^{-1}\right)\left(1+2 K^{-1}\right)>1$

$\mathrm{e}$

$$
\mathbb{P}(\text { sobreviver })=\lim _{j \rightarrow \infty} \mathbb{P}\left(A_{j}\right)
$$




\section{Referências Bibliográficas}

[1] Alves, O. S. M. Transição de fase e forma assintótica em um modelo de reação em cadeia. Tese (Doutorado em Estatística) - Instituto de Matemática e Estatística, Universidade de São Paulo, São Paulo, 2001.

[2] Alves, O. S. M., Machado, F. P., Popov, S. Yu. Phase transition for the frog model. Electron. J. Probab. 7 (16), 21 p., 2002.

[3] Alves, O. S. M., Machado, F. P., Popov, S. Yu. The shape theorem for the frog model. Ann. Appl. Probab. 12 (2), 533-546, 2002.

[4] Alves, O. S. M., Machado, F. P., Popov, S. Yu., Ravishankar, K. The shape theorem for the frog model with random initial configuration. Markov Process. Related Fields 7 (4), 525-539, 2001.

[5] Alves, O. S. M., Lebensztayn, E.; Machado, F. P.; Martinez, M. Z. Random walks systems on complete graphs. Bull. Braz. Math. Soc. 37(4), 571-580, 2006.

[6] Fontes, L. R., Machado, F. P., Sarkar, A. The critical probability for the frog model is not a monotonic function of the graph. J. Appl. Probab. 41 (1), 292-298, 2004.

[7] Lebensztayn, E. Um limitante superior para a probabilidade crítica do modelo dos sapos em árvores homogêneas. Tese (Doutorado em Estatística) - Instituto de Matemática e Estatística, Universidade de São Paulo, São Paulo, 2005 .

[8] Lebensztayn, E., Machado, F. P., Popov, S. An improved upper bound for the critical probability of the frog model on homogeneous trees. J. Statist. Phys. 119 (1-2), 331-345, 2005. 
[9] Lebensztayn, E., Machado, F. P., Martinez, M. Z. Self-avoiding random walks on homogeneous trees. Markov Process. Related Fields, 12 (4), 735$745,2006$.

[10] Leichsenring, A. R. Não monotonicidade do parâmetro crítico no modelo dos sapos. Dissertação (Mestrado em Estatística) - Instituto de Matemática e Estatística, Universidade de São Paulo, São Paulo, 2003.

[11] Popov, S. Yu. Frogs and some other interacting random walks models. In: Discrete Random Walks, DRW'03, Paris, 2003. Discrete Mathematics and Theoretical Computer Science Proceedings. Paris: DMTCS, 2003. AC, 277288.

[12] Popov, S. Yu. Frogs in random environment. J. Statist. Phys. $102(1 / 2)$, 191-201, 2001.

[13] P. Bremaud. Markov chains. Gibbs fields, Monte Carlo simulation, and queues. Texts in Applied Mathematics, 31. Springer-Verlag, New York (1999). 